\title{
Controlled Polymer Desorption from Layer-by-Layer Assembly into Dilute Ionic Solutions
}

\author{
Takeshi SerizAWA, Takehiko OHMORI, and Mitsuru AKASHI ${ }^{\dagger, \dagger \dagger}$ \\ Department of Nanostructured and Advanced Materials, Graduate School of Science and Engineering, \\ Kagoshima University, Korimoto 1-21-40, Kagoshima 890-0065, Japan
}

(Received June 17, 2003; Accepted July 31, 2003)

KEY WORDS Layer-by-Layer Assembly / Desorption / Ionic Solution / Charge Density /

The preparation of ultrathin polymer films on material surfaces not only demonstrates functional modification of the surfaces for potential technological and biomedical applications but also contributes to the investigation of novel polymer characteristics in/on the films. Layer-by-layer (LbL) assembly is a recently developed technique for the preparation of ultrathin polyelectrolyte multilayers on solid substrates, based on alternate immersion of the substrates into aqueous solutions of oppositely charged polyelectrolytes, forming sequential polyion complexes on the substrate surface. ${ }^{1-3}$ Although it is occasionally difficult to prepare structurally regulated films of water-soluble polyelectrolytes by using conventional methods, LbL assembly readily forms regularly layered nanostructures.

Desorption of constituent polymers from LbL assemblies into solutions is important to demonstrate time-controlled release of functional polymers or other molecules from the substrates, and to understand the stability of the assemblies in various environments. Several research groups have investigated desorption of the constituent polymers into aqueous solutions of suitable $\mathrm{pHs}^{4-6}$ and high salt concentrations, ${ }^{6-8}$ breaking of hydrogen bonds and electrostatic interactions formed by weak polyacids and polyamines, respectively. Enzymatic degradation of LbL assemblies composed of naturally occurring polymers has also demonstrated timecontrolled desorption of the polymers., 10 Desorption of polymers under mild conditions has also been triggered by spontaneous hydrolysis of the assembled polymers. ${ }^{11}$ However, fundamental investigation on desorption of constituent polymers from LbL assemblies is still remained. Controlled desorption of LbL assembly triggered by physicochemical stimuli will potenti- ate novel applications in the field of LbL assembly. In the present study, desorption of polymers from LbL assemblies into aqueous ionic solutions, dependent on the chemical structure of the polymers and the ionic concentration, is demonstrated to achieve controlled polymer desorption. A decrease in charge densities of constituent polymers potentially reduced the strength of electrostatic interactions in $\mathrm{LbL}$ assemblies, followed by facile polymer desorption. The assembly and desorption processes were quantitatively analyzed using a quartz crystal microbalance (QCM) substrate. This study demonstrates the possibility that controlled deconstruction of LbL assemblies can be achieved under physiological conditions.

Several polymer combinations were selected for LbL assembly, in order to investigate desorption of polymers formed with different assembly manners. The combinations between cationic poly(vinylamine hydrochloride- $c o-N$-vinylformamide)s [poly(VAm-co$\mathrm{NVF}$ )s], of which the VAm content was readily variable by alkaline hydrolysis of polyNVF, ${ }^{12,13}$ and anionic poly(acrylic acid) (PAAc) were mainly utilized, since the effect of charge densities can be investigated using these polymers. A previous study of LbL assemblies of poly(VAm-co-NVF)s and poly(sodium styrenesulfonate) (PSS) have demonstrated that the thickness was maximal for the assembly of poly(VAm-coNVF) with a VAm content of approximately $30 \%$, indicating that the globular poly(VAm-co-NVF) with a smaller charge density produced an apparently greater thickness at each assembly step. ${ }^{14}$ The thickness was also maximal for the assembly between poly(VAm-coNVF)s with a VAm content of approximately $40 \%$ and PAAc. ${ }^{15}$ Combinations of PAAc or poly(sodium acry-

\footnotetext{
${ }^{\dagger}$ To whom correspondence should be addressed.

${ }^{\dagger}$ Present Address: Department of Molecular Chemistry, Graduate School of Engineering, Osaka University, Yamada-oka, Suita 565-0871, Japan.
} 
late) (PAAc $\cdot \mathrm{Na}$ ) and poly(vinylamine hydrochloride) (PVAm) were also adopted. Since PAAc was dissolved in pure water, the carboxyl groups are partially protonated, indicating that PAAc had a more coiled conformation, compared to PAAc.Na. In fact, the thickness of the former LbL assembly was greater than that of the latter, since the charge density of PAAc in water is smaller than that of PAAc.Na. Other combinations were also selected as references.

\section{EXPERIMENTAL}

\section{Materials}

PAAc, PAAc.Na, and PSS with respective mean weight molecular weights $\left(M_{\mathrm{w}}\right)$ of 2000,2100 , and 70000 were purchased from Aldrich, and were used without further purification. PVAm and poly(VAmco-NVF) were synthesized by alkaline hydrolysis of polyNVF with a mean number molecular weight $\left(M_{\mathrm{n}}\right)$ of $15000\left(M_{\mathrm{w}} / M_{\mathrm{n}}, 3.2\right)$, following methods used in previous papers. ${ }^{12,13}$

\section{Assembly}

A $9 \mathrm{MHz}$ QCM plate with a polished gold electrode [diameter, $0.45 \mathrm{~cm}$; mean roughness, $1.7 \mathrm{~nm}$ ] (USI System, Japan) was used as a substrate for LbL assembly. The assembly was started with cationic polymers. The QCM substrate was immersed into a cationic polymer solution for $20 \mathrm{~min}$ at $25^{\circ} \mathrm{C}$, rinsed with ultra pure water, and then dried with nitrogen gas. The frequency shift was subsequently measured in air, and was converted into the assembly amount by using Sauerbrey's equation: $-\Delta F / \mathrm{Hz}=1.15 \times \Delta m /$ ng. ${ }^{16}$ The substrate was subsequently immersed into an anionic polymer solution under the same conditions, and the same procedure was repeated. The polymer concentration was 0.02 unit $\mathrm{M}$ in all cases. This alternate cycle was repeated for LbL assembly. In all assemblies, approximately $-3000 \mathrm{~Hz}\left(8.2 \mu \mathrm{m} \mathrm{cm}^{-2}\right)$ of the polymers were deposited on the QCM substrate, in order to ignore the effect of the assembly amount. A total of 6, 14, and 18 cycles were necessary to obtain a corresponding amount for the assemblies between poly(VAm-coNVF)s with VAm contents of 36,70 , or $100 \%$ (PVAm), and PAAc, respectively. One hundred cycles were necessary for the assembly of PAAc. Na and PVAm. An almost linear plot of $\Delta F$ against assembly cycle was observed for all assemblies except the assembly of poly(VAm-co-NVF) with a $36 \%$ VAm content and PAAc (the frequency was exponentially shifted in the later ${ }^{15}$ ). The amount ratio between the assembled polymers was approximately 1:1 except the assembly of poly(VAm-co-NVF) with a $36 \%$ VAm content and
PAAc (the assembly amount of PAAc was greater than that of the copolymer).

\section{Desorption}

For desorption experiments, the obtained assembly was immersed into an aqueous ionic solution (mainly, $\mathrm{NaCl}$ ) of suitable concentration and for an adequate time at $25^{\circ} \mathrm{C}$. After immersion, the QCM substrate was rinsed with pure water, and the $\Delta F$ was similarly measured in air. Then, the amount of polymers desorbed was quantified by estimating the decrease in $\Delta m$.

\section{Other Characterization}

Attenuated total reflection (ATR) spectra were obtained with a PerkinElmer Spectrum One (USA) in air at ambient temperature. A QCM electrode with a refractive surface was used to directly observe the ATR spectra. The interferograms were co-added 50 times, and Fourier transformed at a resolution of $4 \mathrm{~cm}^{-1}$. Atomic force microscopic (AFM) images were obtained with a Digital Instruments NanoScope III that was operated in contact mode at ambient temperature after scratching the assemblies with a cantilever. We did not perform any image processing other than flat leveling.

\section{RESULTS AND DISCUSSION}

Figures 1a-1c show desorption percentages of the assemblies between poly(VAm-co-NVF)s with VAm contents of 36,70 , or $100 \%$ (PVAm), and PAAc in aque-

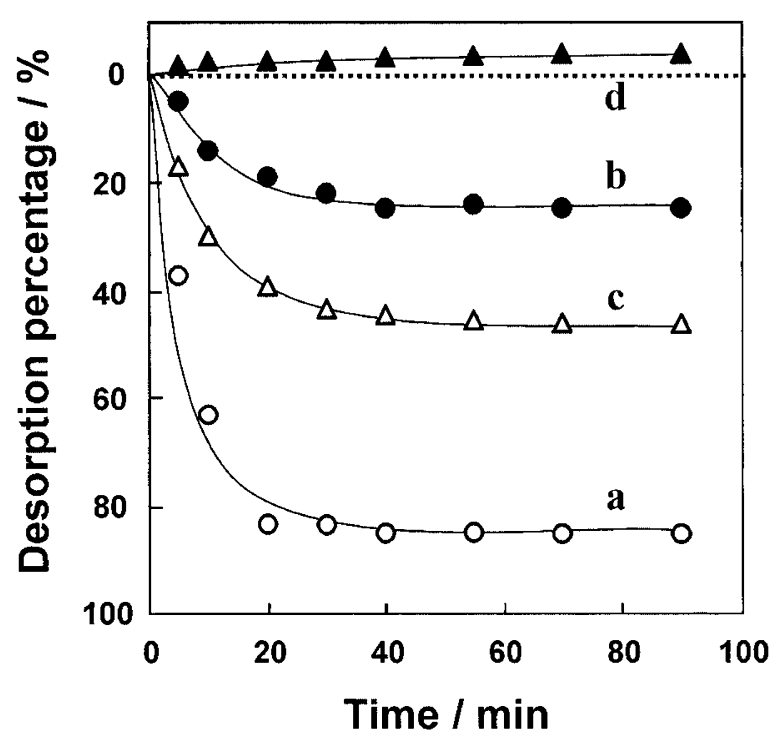

Figure 1. Time-dependent desorption percentages of assemblies of combinations between poly(VAm-co-NVF)s with VAm contents of (a) 36, (b) 70, or (c) 100\% (PVAm), and PAAc, and the combination of (d) PVAm and PAAc. $\mathrm{Na}$ in aqueous $0.1 \mathrm{M} \mathrm{NaCl}$ solution at $25^{\circ} \mathrm{C}$. 
ous $0.1 \mathrm{M} \mathrm{NaCl}$ solution. The polymers assembled were progressively desorbed with increasing immersion time in ionic solutions, possibly due to the penetration of $\mathrm{NaCl}$ into the assemblies and the subsequent relaxation of electrostatic interactions. Approximately $1.5 \mathrm{~h}$ was necessary to obtain equilibrium desorption in all cases. The maximum \% desorption was clearly dependent on the VAm content, and was 85,25 , and $46 \%$ with VAm contents 36,70 , and $100 \%$, respectively. It is difficult to reasonably explain why desorption stopped at a certain percentage, depending on the VAm content. There must be variations in the interactions between the polymers, due to charge distribution in polymers, conformational distribution in the LbL assemblies, and so on. These variations may possibly contribute to the observed differences in \% desorption. There was no linear relationship between the VAm content and the maximum \% desorption percentage. For the assembly of poly(VAm-co-NVF) with a $36 \%$ VAm content and PAAc, the polymers were readily desorbed, since the electrostatic interactions between the polymers seemed to be weaker than in the other polymers. However, the assembly of poly(VAmco-NVF) with a $70 \%$ VAm content was more stable in the ionic environment than that of PVAm, even though the charge density of poly(VAm-co-NVF) was smaller than that of the latter. A similar ordering was observed when the assemblies were desorbed in aqueous $0.01 \mathrm{M} \mathrm{NaCl}$ solution, although the maximum \% desorption was decreased in all cases. At $70 \%$ VAm, the greater number of polymer molecules seemed to cause the polymers to entangle, effectively suppressing attack by $\mathrm{NaCl}$. Klitzing et al. demonstrated LbL assembly between copolymers of diallyldimethylammonium chloride (DADMAC) and $N$-methyl- $N$-vinylacetamide (NMVA) with variable cationic charge densities, and PSS in the presence of $0.1 \mathrm{M} \mathrm{NaCl} .{ }^{17}$ Film thickness after a 10-cycle assembly was steeply increased when the DADMAC content was increased from $50 \%$ to $75 \%$, and was decreased slightly when the cationic fraction exceeded $75 \%$. Caruso et al. also demonstrated that an assembly of the same combinations at a higher ionic strength preferentially occurred at $75 \%$ DADMAC. ${ }^{18}$ Although the experimental systems were different from that of the present study, these observations suggest that a stable assembly can be obtained from copolymers with a $75 \%$ charge density. Since the present study used a weak polyacid, PAAc dissolved in water, the threshold charge density might be smaller due to the fact that the charge density of PAAc is smaller than that of a strong polyacid, such as PSS. It is notable that greater $\mathrm{NaCl}$ concentrations were necessary for desorption of LbL assemblies of poly(VAm-co-NVF)s

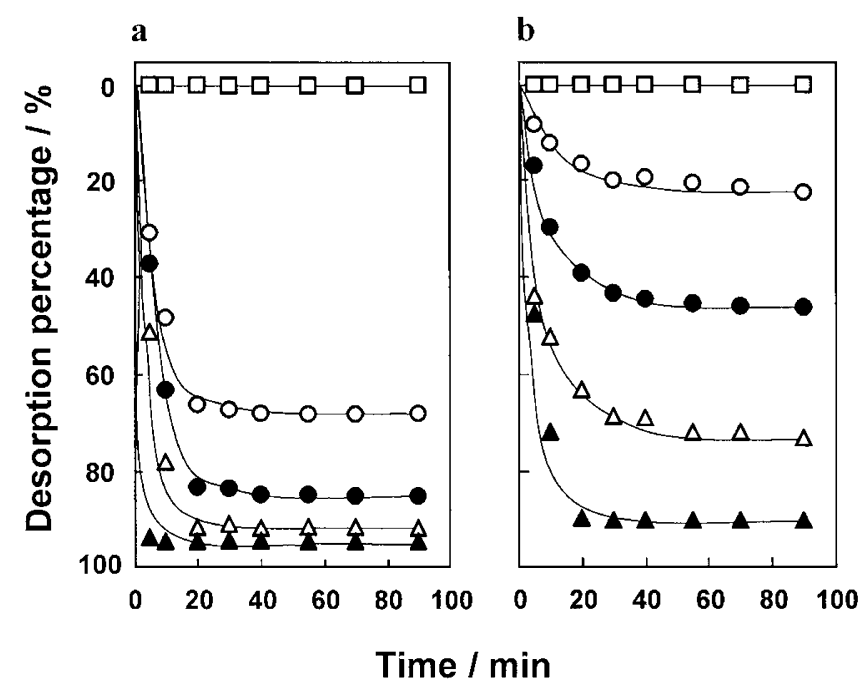

Figure 2. Time-dependent desorption percentages of assemblies consisting of combinations between (a) poly(VAm-co-NVF) with a $36 \%$ VAm content or (b) PVAm, and PAAc in aqueous $\mathrm{NaCl}$ solutions of various concentrations at $25^{\circ} \mathrm{C}$. Open squares, open circles, closed circles, open triangles, and closed triangles indicate desorption in $\mathrm{NaCl}$ solutions of $0 \mathrm{M}, 0.01 \mathrm{M}, 0.1 \mathrm{M}, 1.0 \mathrm{M}$, and $2.0 \mathrm{M}$, respectively.

with various VAm contents and PSS, although the effect of charge density was similar to that observed in the present study. As a consequence, desorption of LbL assemblies could be controlled by altering the charge density of the copolymers used.

Figures $1 \mathrm{c}$ and $1 \mathrm{~d}$ show the effect of the charge density of weak polyacids on desorption in aqueous $0.1 \mathrm{M}$ $\mathrm{NaCl}$ solution. Although the assembly of PAAc was desorbed readily as described above, that of PAAc. Na did not and only swelled with small amounts of $\mathrm{NaCl}$ and water molecules. Since PAAc and PAAc.Na were dissolved in pure water, producing solutions with a $\mathrm{pH}$ of 3.9 and 7.8, respectively, the charge density of PAAc was smaller than that of PAAc.Na. Accordingly, the former assembly, which formed with less ionic interaction with PVAm seemed to be more readily desorbed into the ionic solution. In other words, the presence of counter ions for weak polyacids strongly affects desorption in ionic environments, and assemblies prepared by those weak polyacids are stable in ionic environments. As such, ionic structure is a necessary consideration in the controlled desorption of $\mathrm{LbL}$ assemblies.

It is significant to note that controlled desorption of polymers from LbL assemblies can be achieved under variable ionic conditions. Figure 2 shows desorption profiles of the assemblies between poly(VAm-co-NVF) with a $36 \%$ VAm content or PVAm, and PAAc at $25^{\circ} \mathrm{C}$ in aqueous $\mathrm{NaCl}$ solutions of 0-2.0 M. Initial desorption rates and maximum $\%$ desorption were dependent on the $\mathrm{NaCl}$ concentration, and increased with increasing concentrations of $\mathrm{NaCl}$. The former assembly was 
readily desorbed compared to the latter at all concentrations, possibly due to fewer electrostatic interactions, as described previously. In other words, the former assembly has the potential for rapid desorption of polymers at lower ionic concentrations. In fact, stepwise desorption could be generated by immersion of assemblies into lower ionic strength solutions and subsequently into solutions of greater ionic strength. Not only the charge density of polymers but also the ionic concentration controlled the desorption of polymers from $\mathrm{LbL}$ assembly.

The ATR spectra of the assembly of poly(VAm-coNVF) with a $36 \%$ VAm content and PAAc were obtained at desorption percentages of $0,35,63$, and $85 \%$ (saturation) in aqueous $0.1 \mathrm{M} \mathrm{NaCl}$ solution. Typical peaks of quaternary amine, amide I, and amide II bands were observed for the assembly before desorption at 3216,1668 , and $1541 \mathrm{~cm}^{-1}$, respectively, indicating the presence of the poly(VAm-co-NVF). A carbonyl vibration band of carboxyl groups was also observed at $1714 \mathrm{~cm}^{-1}$, indicating the presence of PAAc. After desorption, the typical peak for PAAc became relatively small compared to the peaks for the copolymer, although both peaks were reduced in size. These observations strongly suggested that greater amounts of poly(VAm-co-NVF) were desorbed from the assembly. Time-dependent ATR spectra of the assembly of PVAm and PAAc similarly demonstrated preferential desorption of PAAc.

AFM observation also demonstrated time-dependent variations in the surface roughness and apparent swelling ratio of assemblies. The assembly of poly(VAm-co-NVF) with a 32\% VAm content and PAAc was used for the AFM observation in air, and the assembly process and the desorption data in aqueous $2.0 \mathrm{M} \mathrm{NaCl}$ solution were similar to those obtained from the copolymer with $36 \% \mathrm{VAm}$. The initial mean roughness $(\mathrm{Ra})$ was $4.5 \mathrm{~nm}$. At a desorption percentage of $40 \%$, the Ra was enlarged $(\mathrm{Ra}=7.9 \mathrm{~nm})$. After the subsequent desorption, the Ra was gradually decreased (desorption percentage, Ra: 60\%, $5.5 \mathrm{~nm} ; 80 \%, 3.7 \mathrm{~nm}$; $95 \%, 1.8 \mathrm{~nm})$, and was reached to that of the QCM substrate $(\mathrm{Ra}=1.7 \mathrm{~nm})$. The assembly with the desorption percentages less than $40 \%$ possibly swelled with $\mathrm{NaCl}$ and water molecules, resulting in the greater Ra. In fact, the apparent swelling ratio of the assembly, which was estimated from the difference in the thickness of the assembly in air and water, was maximal at $40 \%$ desorption, and was decreased with increasing the desorption percentage, supporting the observed changes in the Ra.

Our preliminary experiments utilizing aqueous $\mathrm{CaCl}_{2}$ solution for desorption indicated less \% desorption compared to those for $\mathrm{NaCl}$ solutions, possi- bly due to cross-linking of polymers by divalent $\mathrm{Ca}^{2+}$ ions. Ionic species might be a factor in the desorption of polymers. Desorption was also demonstrated in PBS buffer ( $\mathrm{pH} 7.4)$ containing $\mathrm{NaCl}(137 \mathrm{mM})$, $\mathrm{KCl}(0.268 \mathrm{mM}), \mathrm{KH}_{2} \mathrm{PO}_{4}(0.147 \mathrm{mM})$, and $\mathrm{Na}_{2} \mathrm{HPO}_{4}$ $(0.810 \mathrm{mM})$. More than $90 \%$ of the assembly prepared from poly(VAm-co-NVF) with a $36 \%$ VAm content was desorbed after $5 \mathrm{~min}$ immersion, while $22 \%$ and $8 \%$ of the assemblies prepared from poly(VAm-co$\mathrm{NVF}$ ) with a $70 \%$ VAm content and PVAm, respectively, were desorbed after immersion of a longer time. This observation indicates that it is possible to control desorption of LbL assembly under physiological conditions.

In conclusion, desorption of polymers from LbL assemblies between poly(VAm-co-NVF)s with variable VAm contents and PAAc or PAAc. $\mathrm{Na}$ in aqueous $\mathrm{NaCl}$ solution was quantitatively analyzed by using a QCM substrate. Desorption was controlled by the charge density of the polymer, differences in the counter ions, and the $\mathrm{NaCl}$ concentration of the aqueous solution. Ionic species also affected desorption. The present study contributes not only to understanding of the stability of $\mathrm{LbL}$ assemblies in ionic environments but also to potential biomedical applications of $\mathrm{LbL}$ assemblies such as coating for controlled release. Further detailed research is necessary to thoroughly understand desorption of polymers from $\mathrm{LbL}$ assembly.

Acknowledgment. This work was financially supported in part by Grant-in-Aid for Scientific Research in the Priority Area of "Molecular Synchronization for Design of New Materials System" (No. 404/13022258) and by Grant-in-Aid for Scientific Research (No. 851/14780643) from the Ministry of Education, Culture, Sports, Science and Technology, Japan.

\section{REFERENCES}

1. G. Decher, Science, 277, 1232 (1997).

2. Y. Lvov and H. Möhwald, "Protein Architecture: Interfacing Molecular Assemblies and Immobilization Biotechnology", Marcel Dekker, New York, N.Y., 2000.

3. S. Tripathy, J. Kumar, and H. S. Nalwa, Eds., "Handbook of Polyelectrolytes and Their Applications", American Scientific Publishers, Los Angeles, CA, 2002, vol. 1.

4. S. A. Sukhishvili and S. Granick, J. Am. Chem. Soc., 122, 9550 (2000).

5. S. Y. Yang and M. F. Rubner, J. Am. Chem. Soc., 124, 2100 (2002).

6. S. Dubas, T. R. Farhat, and J. B. Schlenoff, J. Am. Chem. Soc., 123, 5368 (2001).

7. S. T. Dubas and J. B. Schlenoff, Macromolecules, 34, 3736 (2001). 
8. C. Schuler and F. Caruso, Biomacromolecules, 2, 921 (2001).

9. T. Serizawa, M. Yamaguchi, and M. Akashi, Macromolecules, 35, 8656 (2002)

10. T. Serizawa, M. Yamaguchi, and M. Akashi, Angew. Chem. Int. Ed., 42, 1115 (2003).

11. E. Vázquez, D. M. Dewitt, P. T. Hammond, and D. M. Lynn, J. Am. Chem. Soc., 124, 13992 (2002).

12. K. Yamamoto, T. Serizawa, Y. Muraoka, and M. Akashi, $J$. Polym. Sci., Part A: Polym. Chem., 38, 3674 (2000).

13. K. Yamamoto, T. Serizawa, Y. Muraoka, and M. Akashi,
Macromolecules, 34, 8014 (2001).

14. T. Serizawa, N. Kawanishi, and M. Akashi, Macromolecules, 36, 1697 (2003).

15. T. Serizawa, N. Kawanishi, and M. Akashi, submitted.

16. G. Sauerbrey, Z. Phys., 155, 206 (1959).

17. R. Steitz, W. Jaeger, and R. v. Klitzing, Langmuir, 17, 4471 (2001).

18. B. Schoeler, G. Kumaraswamy, and F. Caruso, Macromolecules, 35, 889 (2002). 